\title{
MAXIMAL FUNCTION ON GENERALIZED LEBESGUE SPACES $L^{p(\cdot)}$
}

\author{
L. DIENING
}

Abstract. We prove the boundedness of the Hardy-Littlewood maximal function on the generalized Lebesgue space $L^{p(\cdot)}\left(\mathbb{R}^{d}\right)$ under a continuity assumption on $p$ that is weaker than uniform Hölder continuity. We deduce continuity of mollifying sequences and density of $C^{\infty}(\bar{\Omega})$ in $W^{1, p(\cdot)}(\Omega)$.

Mathematics subject classification (2000): 42B25, 46E30.

Key words and phrases: maximal function, generalized Lebesgue spaces, generalized Orlicz spaces, mollifier, electrorheological fluids.

\section{REFERENCES}

[1] EMILIO ACERBI AND GIUSEPPE MingIONE, Regularity results for a class of functionals with non-standard growth, Arch. Ration. Mech. Anal. 156 (2001), no. 2, 121-140.

[2] JÖRAN BERGH AND JÖRGEN LÖFSTRÖM, Interpolation spaces. An introduction, Springer-Verlag, Berlin, 1976, Grundlehren der Mathematischen Wissenschaften, No. 223.

[3] LARS DIENING, Theoretical and numerical results for electrorheological fluids, Ph.D. thesis, University of Freiburg, Germany, 2002.

[4] DAVId E. EDMUndS AND JIŘí RÁKOSNÍK, Density of smooth functions in $W^{k, p(x)}(\Omega)$, Proc. Roy. Soc. London Ser. A 437 (1992), no. 1899, 229-236.

[5] Sobolev embeddings with variable exponent, Studia Math. 143 (2000), no. 3, 267-293.

[6] LAWRENCE C. EVANS AND RONALD F. GARIEPY, Measure theory and fine properties of functions, CRC Press, Boca Raton, FL, 1992.

[7] HENRYK HUDZIK, The problems of separability, duality, reflexivity and of comparison for generalized Orlicz-Sobolev spaces $W_{M}^{k}(\Omega)$, Comment. Math. Prace Mat. 21 (1980), no. 2, 315-324.

[8] ONDREJ KovÁČIK AND JiŘí RÁKOSNÍK, On spaces $L^{p(x)}$ and $W^{k, p(x)}$, Czechoslovak Math. J. 41(116) (1991), no. 4, 592-618.

[9] PAOLO MARCELLINI, Regularity and existence of solutions of elliptic equations with p, $q$-growth conditions, J. Differential Equations 90 (1991), no. 1, 1-30.

[10] J. MusieŁaK AND W. ORLICZ, On modular spaces, Studia Math. 18 (1959), 49-65.

[11] Julian MusieŁAK, Orlicz spaces and modular spaces, Springer-Verlag, Berlin, 1983.

[12] Luboš PicK AND MichaEL RŮŽIČKA, An example of a space $L^{p(x)}$ on which the Hardy-Littlewood maximal operator is not bounded, Expo. Math. 19 (2001), no. 4, 369-371.

[13] MICHAEL RŮŽIČKA, Electrorheological fluids: modeling and mathematical theory, Springer-Verlag, Berlin, 2000.

[14] S. G. SAmKo, Density $C_{0}^{\infty}\left(\mathbf{R}^{n}\right)$ in the generalized Sobolev spaces $W^{m, p(x)}\left(\mathbf{R}^{n}\right)$, Dokl. Akad. Nauk 369 (1999), no. 4, 451-454.

[15] Stefan G. SAmKo, Convolution and potential type operators in $L^{p(x)}\left(\mathbf{R}^{n}\right)$, Integral Transform. Spec. Funct. 7 (1998), no. 3-4, 261-284.

[16] Elias M. STEIN, Singular integrals and differentiability properties of functions, Princeton University Press, Princeton, N.J., 1970.

[17] V. V. ZHIKOV, Averaging of functionals of the calculus of variations and elasticity theory, Izv. Akad. Nauk SSSR Ser. Mat. 50 (1986), no. 4, 675-710, 877.

[18] Meyer-type estimates for solving the nonlinear Stokes system, Differ. Uravn. 33 (1997), no. 1, $107-114,143$.

[19] VASILII V. Zhikov, On Lavrentiev’s phenomenon, Russian J. Math. Phys. 3 (1995), no. 2, $249-269$. 